\title{
Case Report \\ Paediatric Stroke: Review of the Literature and Possible Treatment Options, including Endovascular Approach
}

\author{
Elisa F. Ciceri, ${ }^{1}$ Valeria Cuccarini, ${ }^{1}$ Luisa Chiapparini, ${ }^{1}$ Veronica Saletti, ${ }^{2}$ \\ and Luca Valvassori ${ }^{3}$
}

${ }^{1}$ Neuroradiology Department, Fondazione Istituto “C. Besta”, Via Celoria 11, 20133 Milan, Italy
${ }^{2}$ Paediatric Department, Fondazione Istituto "C. Besta", Via Celoria 11, 20133 Milan, Italy
${ }^{3}$ Neuroradiology Department, Ospedale Niguarda, Piazza Ospedale Maggiore 3, 20162 Milan, Italy

Correspondence should be addressed to Elisa F. Ciceri, elisaciceri@yahoo.com

Received 23 September 2010; Revised 11 May 2011; Accepted 12 May 2011

Academic Editor: Halvor Naess

Copyright (c) 2011 Elisa F. Ciceri et al. This is an open access article distributed under the Creative Commons Attribution License, which permits unrestricted use, distribution, and reproduction in any medium, provided the original work is properly cited.

\begin{abstract}
Stroke is among the top 10 causes of death in childhood. More than half of the surviving children have long-term neurological sequelae. Ischemic stroke (IS) includes arterial ischemic stroke and cerebral venous thrombosis with venous infarction. Haemorrhagic stroke (HS) includes intracerebral haematoma or subarachnoid haemorrhage. Risk factors for stroke are different in children and in adults. 10-30\% of IS have no identified risk factors. However, multiple risk factors are recognizable in the majority of stroke in children; thus, a comprehensive diagnostic evaluation is crucial. Vascular abnormalities, such as arteriovenous malformations, aneurysms, vessel dissection, stenosis, and moyamoya disease, are frequently associated with both IS and HS and lead to high recurrence rates. Endovascular and surgical treatment options are sometimes indicated, performed on the basis of expert opinion, and extrapolated from the adult procedures. In the present paper, we review the recent literature and we discuss the treatment in five cases managed at our institutions.
\end{abstract}

\section{Introduction}

In young population stroke is often an underestimated cause of neurological disturbances with different possible aetiologies and multiple treatment options. A thorough knowledge of the pathophysiology of the disease, of the natural history and of the possible different therapies including medical or surgical approaches, represents a necessary instrument in order to obtain a prompt and correct diagnosis and a successful recovery. Additionally, diagnosis of paediatric stroke is even more difficult, due to the limited expressive and interpretive skills of symptoms compared to the adult population.

Stroke is among the top 10 causes of death in childhood with an incidence of $2-13$ per 100,000 children. More than half of the surviving children who had a stroke have longterm neurological sequelae [1].

Ischemic stroke (IS) refers to both arterial ischemic stroke (AIS) and cerebral venous thrombosis (CVT) resulting in venous infarction. Hemorrhagic stroke (HS) includes intracerebral haemorrhage and nontraumatic subarachnoid haemorrhage. Nearly half of paediatric strokes are hemorrhagic [2].

There are differences in the incidence of stroke during childhood, with incidence rate of 3/100,000 under the age of 1 year (even when neonatal or perinatal stroke, which is known to have a particularly high incidence rate, is excluded) and of 0.7/100,000 at 5-9 years. Another incidence peak is observed in male adolescents probably due to hormonal factors [3].

The risk factors for childhood stroke are markedly different from those in adults, including young adults. Childhood stroke in which no risk factors are identified represent $10-30 \%$ of cases [3].

In the next paragraphs we try to give a schematic presentation of the main risk factors for arterial, venous, and haemorrhagic stroke, an overview about the stroke outcome, and of the recurrence rate. In evaluating this complex pathology, it is always important to consider that the same risk factor can determine all the three situations and 
that multiple factors can differently combine each others in the same situation.

\section{Risk Factors}

\subsection{Arterial Ischemic Stroke (AIS)}

2.1.1. Arteriopathies. In childhood AIS, abnormalities of the cerebral circulation (both intracranial and cervical) have been reported in up to $80 \%$ of the cases [3]. The most frequently identified form of arteriopathy is termed transient cerebral arteriopathy (TCA) or focal cerebral arteriopathy of childhood (FCA), defined as vascular stenosis not otherwise classified as dissection, moyamoya, sickle cell arteriopathy (SCA), postvaricella arteriopathy, vasculitis, or other specific diagnoses. TCA or FCA represents a monophasic transient arteriopathy affecting large- or medium-sized vessels that, on follow-up imaging few months after the infarct, generally shows regression or nonprogression. It seems that many cases of FCA have a postinfectious mechanism underlying the arteriopathy, particularly recent upper respiratory tract infections [3].

Craniocervical arterial dissection may be responsible for 7 to $20 \%$ of childhood AIS. In children, the anterior circulation is involved more frequently than the posterior circulation. There is a history of trauma in half of the cases although the trauma may be minor; in these cases the cervical arteries are most commonly affected, while intracranial dissection accounts for at least a quarter of the cases. In nontraumatic cases $60 \%$ of dissections are intracranial. In dissecting diseases, symptoms can be related either to mass effect, ischemia, or SAH. If all vascular layers of the intradural artery are dissected, a subarachnoid hemorrhagic will occur. Intramural clot formation can cause distal thromboembolic ischemia or occlusion of perforator branches and local ischemia. Chronic dissecting process may eventually lead to formation of a "giant partially thrombosed aneurysm" [4]. Unlike in the adult, an underlying condition predisposing to dissection is seldom found in children. Recurrence rate is about $10 \%[1,3,5]$.

Moyamoya is a noninflammatory nonatherosclerotic vasculopathy. It is characterized by progressive occlusion or stenosis of the cerebral vasculature particularly involving the vessels feeding the circle of Willis, most commonly of the distal internal carotid arteries, with compensation resulting in the development of an extensive network of hypertrophic arterial collaterals distal to the occlusion. Moyamoya can be idiopathic (disease) or secondary (syndrome) when associated with other causes of vasculopathy, such as sickle cell disease (SCD), Down's syndrome, neurofibromatosis, and previous cranial irradiation. Genetic polymorphisms of HLA class II genes have been associated with idiopathic moyamoya disease $[6,7]$.

Primary CNS vasculitis or systemic vasculitis (lupus erythematosus, Henoch-Schonlein purpura, Wegener granulomatosis, polyarteritis nodosa, Takayasu arteritis, and Kawasaki disease) have been linked to stroke.

Rare causes of cerebral arteriopathy in children include neurofibromatosis 1 (NF1) arterial dysplasia, postirradiation arteriopathy, fibromuscular dysplasia, and collagen disorders.

2.1.2. Cardiac Diseases. Cardiac disease is one of the most commonly identified risk factors in childhood IS, as it is identified in $15-30 \%$ of cases of AIS.

In children with preexisting major congenital cardiac malformations, embolic phenomena are the major cause of stroke. Other factors involved include hypoxia, hypotension, and polycytemia.

An association between IS and cardiac surgery or catheterization is found in about $40 \%$ of children with cardiac disease.

Patent foramen ovale (PFO) has been referred to as "the back door to the brain" through which small venous thrombi not filtered by the pulmonary vasculature enter the systemic circulation. In children and young adults with otherwise cryptogenic stroke, an increased prevalence of PFO has been found, even if there are not conclusive data demonstrating causality [8].

Children with AIS may have both cardiac disease and intracranial arteriopathy.

2.1.3. Haematological Disorders. In children with SCD the risk of stroke is about 200 times greater than in other children, and up to one-third of children with SCD have silent strokes (neuroimaging evidence without history of stroke) that frequently lead to cognitive deficits. $\beta$-thalassemia can also lead to IS.

Both of these conditions can cause stroke because of anemia and hypercoagulable state. Moreover, development of stenosis of the intracranial internal carotid artery (ICA) and of the proximal middle cerebral artery (MCA) in SCD determines a secondary moyamoya like vasculopathy due to repeated insults on the vessels' walls.

Iron-deficiency anaemia has been found to be present in $20 \%$ of previously healthy children with AIS.

A number of genetic thrombophilic traits have been associated with paediatric stroke: prothrombin G20210A, factor V Leiden G1691A, MTHFR C677T, lipoprotein(a), antiphospholipid antibodies/LLAC, protein S deficiency, antithrombin deficiency, protein $\mathrm{C}$ deficiency, different haplotypes of genes involved in the nitric oxide pathways and thus in possible endothelial impairment.

2.1.4. Infections. CNS infections, that is, meningitis and encephalitis, are present in $10 \%$ of cases of AIS, presumably due to vascular inflammation and thrombosis or due to reduced cerebral perfusion secondary to systemic hypotension, raised intracranial pressure, and low cerebrospinal fluid glucose.

Hypotension and hypercoagulable state as a consequence of bacteraemia or sepsis can lead to IS.

Focal cerebral artery stenosis with a history of varicella infection in the preceding year has been termed postvaricella angiopathy, thought to be due to viral invasion of the artery wall as already mentioned in the previous section on arteriopathies. 
The most common risk factor identified in childhood stroke due to CVT is represented by middle-ear and paranasal sinus infections.

2.1.5. Genetics. An increased risk of stroke has been associated with numerous genetic diseases, such as the abovementioned SCD and genetically inherited thrombophilic traits, Down's syndrome, NF1, Williams syndrome, homocystinuria, cerebral autosomal-dominant arteriopathy with subcortical infarcts and leukoencephalopathy (CADASIL) and Fabry disease, mitochondrial encephalomyopathy with lactic acidosis and stroke (MELAS) [9].

AIS and CVT share many of the above-mentioned risk factors.

2.2. Cerebral Sinovenous Thrombosis (CVT). CVT in childhood is a rare (incidence 0.4-0.7 per 100000 children per year, most occurring within the neonatal period) but underrecognized disorder, often of multifactorial aetiology, with neurologic sequelae in up to $40 \%$ of survivors and mortality approaching $10 \%$. The spectrum of brain injury varies from venous congestion to parenchymal ischemia which may be cortical or subcortical and involve deep grey matter. The majority of the parenchymal infarcts are haemorrhagic. Subarachnoid and subdural haemorrhage are less frequent. In neonates there is a strong association between CVT and intraventricular haemorrhage. Infections appear to be the most common condition associated to CVT. Predisposing systemic comorbidity factors include fever, infection, dehydration, anaemia, and chronic medical conditions such as congenital heart disease, nephritic syndrome, autoimmune diseases, malignancy, and genetic and acquired prothrombotic disorders. Moreover, locoregional factors such as first of all otitis media and mastoiditis, then meningitis, head trauma, and recent intracranial surgery are strongly related to CVT [10].

\subsection{Haemorrhagic Stroke (HS)}

2.3.1. Structural Lesions. Structural lesions account for at least half of HS in children. Arteriovenous malformations (AVMs) are the most commonly identified structural lesions found in HS (30\%), and $80 \%$ of the children with an AVM will declare their malformation by HS. Probability of a first haemorrhage is $2-4 \%$ per year [11].

The vast majority of arterial cerebral aneurysms in the paediatric age are symptomatic, and they account for $13 \%$ of HS, mostly presenting with subarachnoid haemorrhage (SAH). Aneurysms may be idiopathic $(30 \%)$ or related to arterial dissection $(50 \%)$, to bacterial or mycotic infections (15\%), or to trauma (5-10\%) [12]. In traumatic cases, HS often presents $2-4$ weeks after the head injury.

HS is also related to SCD (25\% of first strokes in children with SCD are haemorrhagic) and moyamoya, due to fragility of the dysplastic collateral vessels.

Cavernous malformations are found to be the cause of bleeding in $15 \%$ of $\mathrm{HS}$ paediatric cases.

Finally, intratumoral haemorrhage can be a rare feature of intracranial malignancy.
There is a threefold increased incidence of haemorrhage in the posterior circulation in children compared to adults and a fivefold increased incidence in involvement of the internal carotid artery termination $[13,14]$.

2.3.2. Haematological Disorders. Between 10 and $30 \%$ of childhood HS are caused by haematological disorders such as haemophilia, thrombocytopenia, von Willebrand's disease, coagulopathy secondary to hepatic dysfunction, or vitamin K deficiency [3].

About $25 \%$ of children have HS of undetermined cause.

\section{Outcome}

Fatality rates vary from 2 to $20 \%$ for IS and from 5 to $54 \%$ for HS. Male gender, black ethnicity, and stroke recurrence increase the risk of death. Neurological deficits are reported to be present in at least two-thirds of survivors.

Lesion location and extension is an important predictor of outcome.

Even if it is widely believed that brain plasticity can lead to improved outcome following acquired brain injury at an early age, there is increasing evidence to support the hypothesis that younger age at time of stroke is a predictor of a worse outcome, particularly for cognitive and neuropsychological domains $[2,3,15,16]$. Mortality rate in paediatric group is reported to be 6 to $30 \%$ [17]. Therefore, children should be closely monitored over the time for possible recurrences.

These are aspects that should be taken into consideration also in children with silent infarctions.

\section{Recurrence}

Recurrence risk of AIS is about $1.2 \%$ in the perinatal age and $19 \%$ in later infancy and childhood, but probably a greater number of children have TIAs or silent reinfarctions. Fiveyear followup shows no recurrence in children with normal vascular imaging and up to $60 \%$ recurrence rate in children with vascular abnormalities [14].

HS recurrence rate is about $10 \%$ at 5 -year followup. No recurrences are expected in idiopathic HS whereas $13 \%$ recurrence rate in children with vascular structural anomalies and in those with medical causes of HS; in the latter cases, recurrence tends to occur within the first week, while in the former it may occur years later [18].

Between $10 \%$ and $20 \%$ of children who have a CVT will experience a recurrent symptomatic venous event, half of which systemic rather than cerebral [10].

\section{Diagnostic Imaging}

Cerebral imaging includes computed tomography (CT) particularly when HS is suspected in order to rapidly discriminate between HS and IS and to distinguish between intracerebral haematoma or SAH. Magnetic resonance imaging (MRI) with angio-MR (MRA) helps to precisely identify the site of IS/HS, the involved structures, the presence of oedema, and/or mass effects. Diffusion MR (DWI) and 


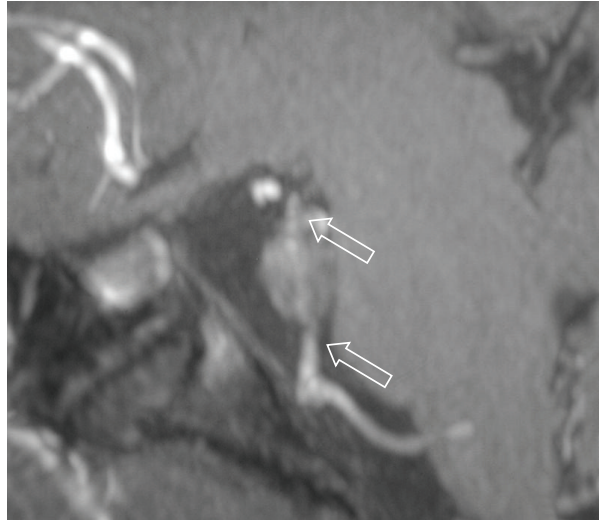

(a)

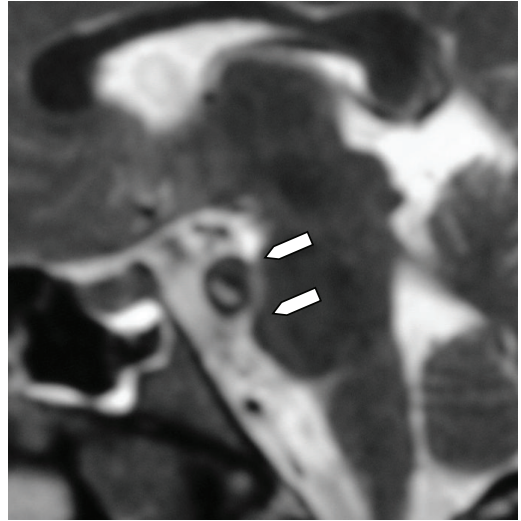

(b)

Figure 1: MRA (a) and MRI sagittal sections (b), obtained 11 days after the initial symptoms, demonstrate the presence of a BA aneurysm with mass effect on the pons (arrowheads in (b)) and ischemic changes (not shown). At both edges of the aneurysm, stenosis of the BA is recognizable (arrows in (a)).

perfusion MR (PWI) are useful to evaluate the timing of the stroke and the cerebral blood flow in the surrounding areas. Gradient-echo and susceptibility-weighted images MR (GRE/SWI) are able to detect deoxyhaemoglobin in very recent bleeding when other sequences are unable to demonstrate the extent of the haematoma or to detect small amount of haemosiderin in old HS or in cryptic malformations, such as cavernous angiomas. Finally, in selected cases, digital subtraction cerebral angiography (DSA) is recommended to better evaluate the vascular tree. Specifically, dedicated cerebrovascular imaging with DSA is crucial when no cause for stroke is found via noninvasive vascular imaging. Sometimes vascular malformations are not immediately evident after the acute haemorrhage; therefore, when vascular imaging is normal or inconclusive, the studies should be repeated once the clot has been reabsorbed $[1,3,14,18]$.

\section{Treatment}

Three consensus papers suggest the guidelines for the evaluation and treatment of AIS in childhood $[15,19,20]$, and one encompasses guidelines for the treatment or evaluation of paediatric HS [20].

6.1. Medical Treatment. Acute and chronic management of IS includes several options, depending on the aetiology of stroke, timing, extent, and so forth. Antioedema and antiplatelet treatments are often indicated. Anticoagulant therapies are usually employed in cardiac cause, CVT, dissection, and recurrent stroke while on antiplatelet therapy. Exchange transfusion (SCD), cerebral and systemic supportive care, and correction of specific conditions are always required. Paediatrics encompasses a wide range of ages with maturational differences. Optimal dosing is likely to differ as there are age-dependent differences in haemostasis, younger individuals demonstrating diminished specific indices of fibrinolysis and global, increased fibrinolytic capability [17].

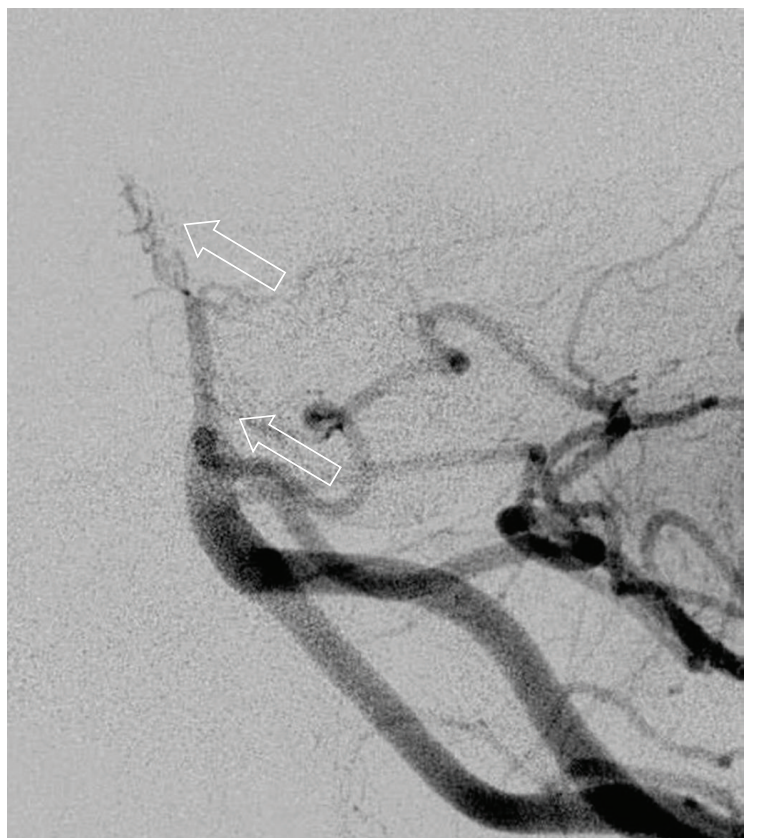

FIGURE 2: Follow-up angiogram, obtained 11 months after the onset of symptoms, shows complete obliteration of the BA at the level of the dissection, without any evidence of residual filling of the aneurysm.

CVT treatment includes general supportive measures and antithrombotic and nonantithrombotic therapies. Anticoagulant and antiplatelet treatment regimens vary between centers, and some of them prefer unfractioned heparin in the acute state, as the effects of heparin can be reversed if intracranial haemorrhage occurs. In the following 3 to 6 months, chronic anticoagulation is maintained with Low Molecular Weight Heparin (LMWH) or Coumadin [10]. Treatment of neonates with LMWH appears to be safe and should at least be considered. There are very few reports 


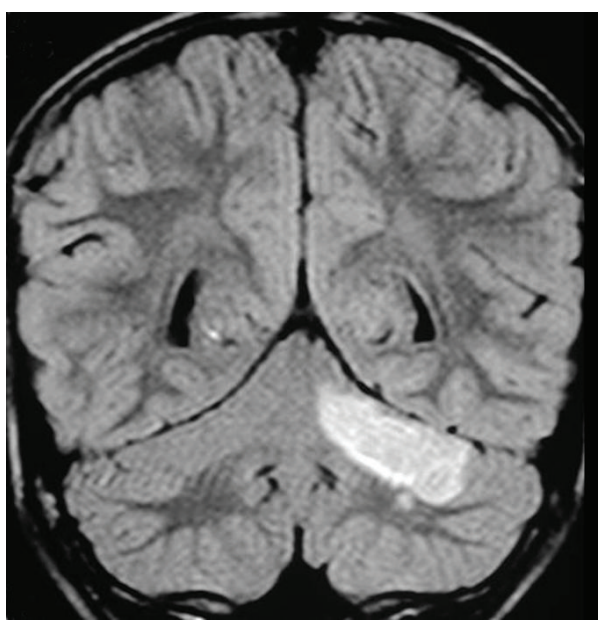

(a)

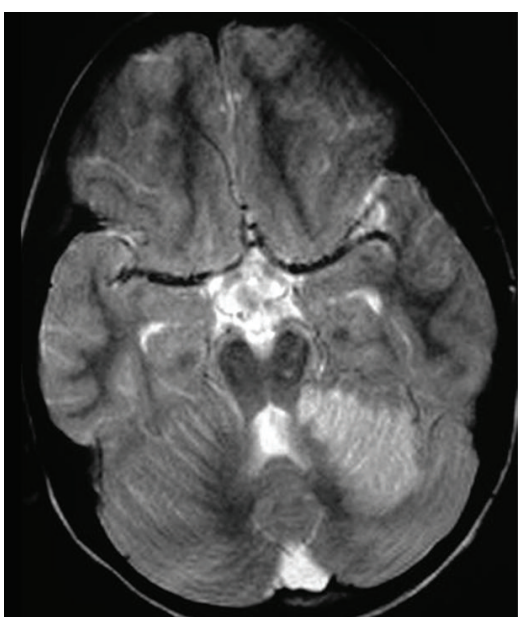

(b)

FIgURE 3: MRI coronal FLAIR (a) and axial T2-weighted (b) images show an infarction in the left superior cerebellar hemisphere, in the territory of one hemispheric branch of the left SCA.

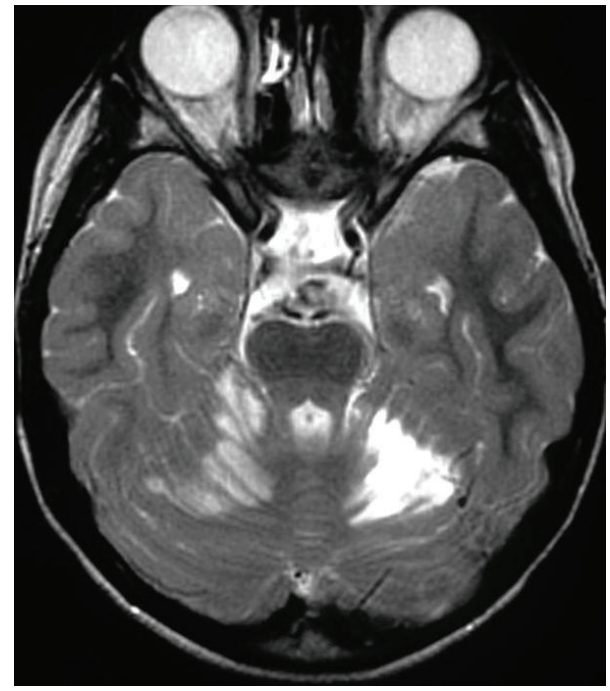

(a)

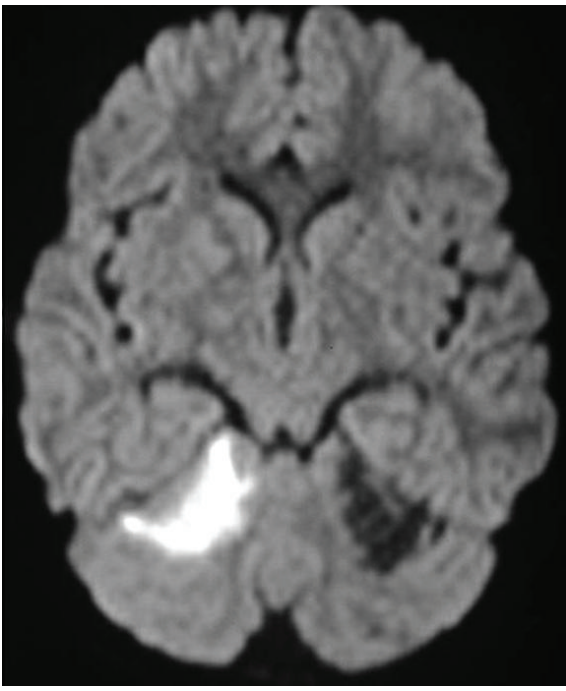

(b)

FIGURE 4: MRI axial T2-weighted (a) and diffusion-weighted (DWI) (b) images demonstrate the old infarction in the left superior cerebellar hemisphere and the new lesion in the right superior cerebellar hemisphere with identical distribution. The restricted diffusivity (high signal intensity in (b)) indicates a recent infarction.

on the use of antiplatelet agents such as acetylsalicylic acid (ASA) or dipyridamole in the acute or chronic settings in paediatric age [10]. If significant ICH is associated with CVT, the use of anticoagulants is controversial. Septic situation requires antibiotics and may need surgical removal and drainage of the infection. Medical treatment of HS is mainly supportive, with correction of treatable haemorrhage risk factors or correction of specific conditions such as, for instance, antimicrobial treatment in infectious aneurysms.

While a neuroprotective role has been ascribed to hypertonic solutions of albumin, memantine $\mathrm{HCl}$, and magnesium sulphate that are recommended for the treatment of adult ischemic stroke patients, no data are available in order to make recommendations regarding use in paediatric AIS [17].

6.2. Surgical Options. Within the management of paediatric stroke, invasive treatments are rarely performed in selected cases, based on expert opinion [11,12,16, 21-27].

Surgical revascularization procedures include direct surgical bypass or indirect procedures to encourage development of collateral blood vessels such as the encephaloduroarteriomyosinangiosis in moyamoya. In case of massive cerebellar oedema or haematoma, decompressive craniectomy and haematoma evacuation can be performed to prevent tonsillar herniation. Decompressive surgery is 


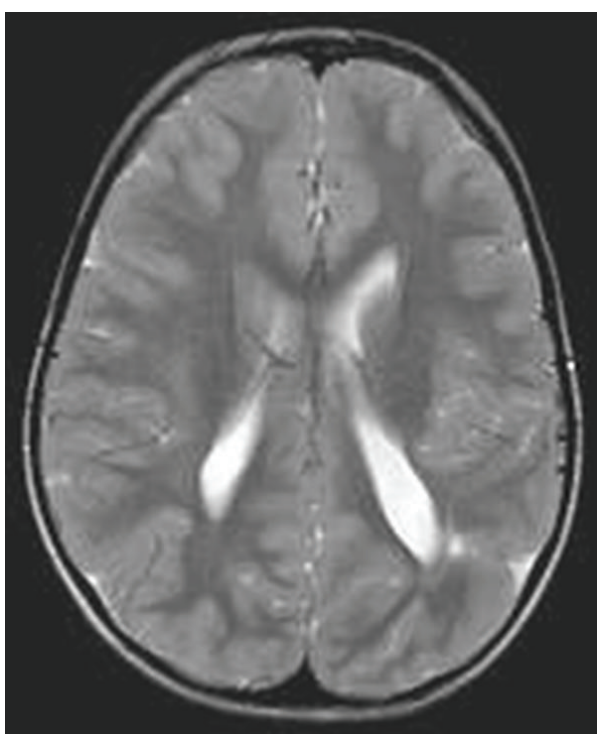

FIGURE 5: MRI axial T2 weighted shows the cortical-subcortical abnormalities in the left temporo-parietal-occipital region.

required in supratentorial large cerebral lesions with mass effect and transtentorial herniation. In hydrocephalus, after intraventricular haemorrhage or SAH, external ventricular drainage may be required. In vascular malformations such as aneurysms or AVMs, open surgery represents a possible treatment.

6.3. Endovascular Options. The endovascular options in case of IS comprise (1) intra-arterial thrombolysis and/or mechanical revascularization in thromboembolic lesions, (2) revascularization procedures with stenting with or without angioplasty in stenoses or dissections, and (3) intra-arterial administration of vasodilator drugs in vessels spasm. In cases of HS due to bleeding from vascular malformations, endovascular repair with platinum detachable coils or flow diverter for aneurysms, and histoacrylic or polymeric glue injections in AVM are possible alternatives. In selected cases, surgical and endovascular approaches can be combined. An additional option for the treatment of small AVM is represented by radiosurgery [28]. In case of cardiac disease (i.e., PFO), correction of the cardiac defect should be considered.

\section{Illustrative Cases}

7.1. Case 1 (Figures 1 and 2). A 15-year-old female patient was admitted to our hospital for angiographic followup and possible treatment of a partially thrombosed basilar artery (BA) dissecting aneurysm extending from the midbasilar portion to the origin of the superior cerebellar arteries (SCAs). One week before her first hospitalization the patient had complained of sudden nuchal headaches sometimes associated with nausea, vomiting, and visual changes, which disappeared without any treatment. No history of previous trauma was reported. Physical and neurological examinations were within normal limits. Four-vessel cerebral digital subtraction angiography (DSA) and MRA (Figure 1) demonstrated the presence of the BA aneurysmal dilatation measuring $5 \mathrm{~mm}$ in its patent portion with an extension of $2 \mathrm{~cm}$ in length. Stenosis of the BA was recognizable proximal and distal to the aneurysm (Figure 1). Both SCAs were injected from the BA. MR imaging also visualized small ischemic areas in the left lower part of the pons and in the left cerebral peduncle (Figure 1). The patient was discharged on antiplatelet therapy (ticlopidine $250 \mathrm{mg} / \mathrm{dL}$ ) to prevent further thrombotic events. The 2- and 4-month followup MR imaging with MRA showed further progression of the BA stenosis. Due to the good clinical condition of the patient, endovascular revascularization was not performed because of the high procedural risks. Finally, the follow-up angiogram (Figure 2) obtained 11 months after the initial symptoms demonstrated complete obliteration of the BA at the level of the dissection (arrows) without any evidence of filling of the aneurysmal pouch and adequate collateral flow to the upper BA, posterior cerebral arteries (PCAs), and SCAs provided by the internal carotid arteries via the posterior communicating arteries. MR imaging performed at the same time demonstrated shrinkage of the aneurysm without new ischemic lesions. The 19-month follow-up MR imaging ruled out the presence of new ischemic areas. At the present time, 6 years later, the patient remains clinically stable and neurologically intact. The extensive workup for suspected collagenopathy, including genetic research for the type 4 Ehlers-Danlos and collagen biopsy, was negative.

7.2. Case 2 (Figures 3 and 4). A 7-year-old roller-skater male was first admitted at a primary medical center with a 4hour history of acute onset of unstable gait and left-limb weakness during a play fight with his sister, followed by diffuse headache. A brain CT scan was normal. Over the next 12 hours the patient's balance disorder improved, while left-limb weakness remained stable and a mild left cerebellar syndrome. Brain MRI and magnetic resonance angiography (MRA) showed a left cerebellar ischemic stroke and absence of the left superior cerebellar artery (SCA). Cardiac disorders were initially excluded by transthoracic echocardiogram.

The patient was admitted to our institute 5 days after symptoms onset. Anti-platelet treatment was started. Head and neck angio-CT and brain MRI revealed an infarction in the left superior cerebellar hemisphere (Figure 3 ) and the presence of a possible dissection of the left vertebral artery close to the foramen magnum. The cerebral DSA showed a lumen margin irregularity consistent with a fresh thrombus in the proximal tract of the left SCA. Laboratory findings excluded haematological disorders such as thrombophilias and SCD.

Over the next 7 days the patient's neurological symptoms gradually improved. Then, one week later, he abruptly developed headache, repeated vomiting, disturbance of consciousness with drowsiness, and right limb weakness. Brain MRI demonstrated a new cerebellar ischemic lesion in the superior right cerebellar hemisphere (Figure 4). Anticoagulant medication (low molecular weight heparin) was initiated. Over the following days the patient had 


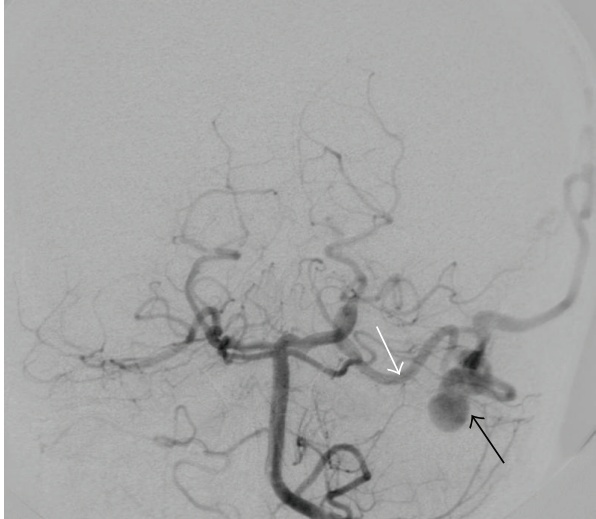

(a)

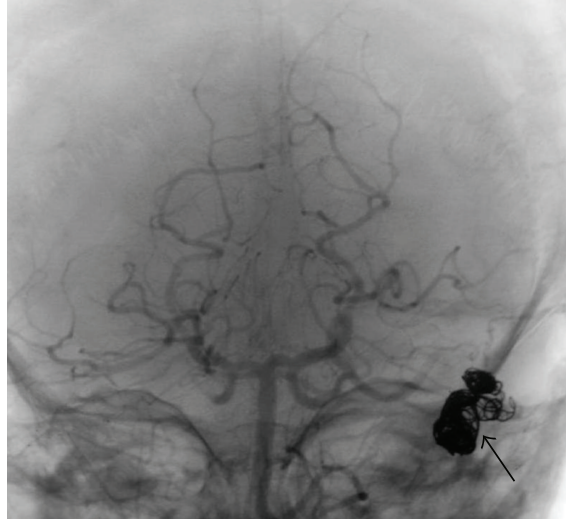

(b)

FIGURE 6: Anteroposterior view of the left vertebral angiography showing a direct arteriovenous shunt between the anterior temporal branch of the left PCA (white arrow) and a superficial temporal vein with an interposed irregular aneurysmal dilatation (black arrow in (a)). The posttreatment angiography (b) demonstrates the complete resolution of the shunt obtained utilizing detachable platinum coils and histoacrylic glue (arrow in (b)).

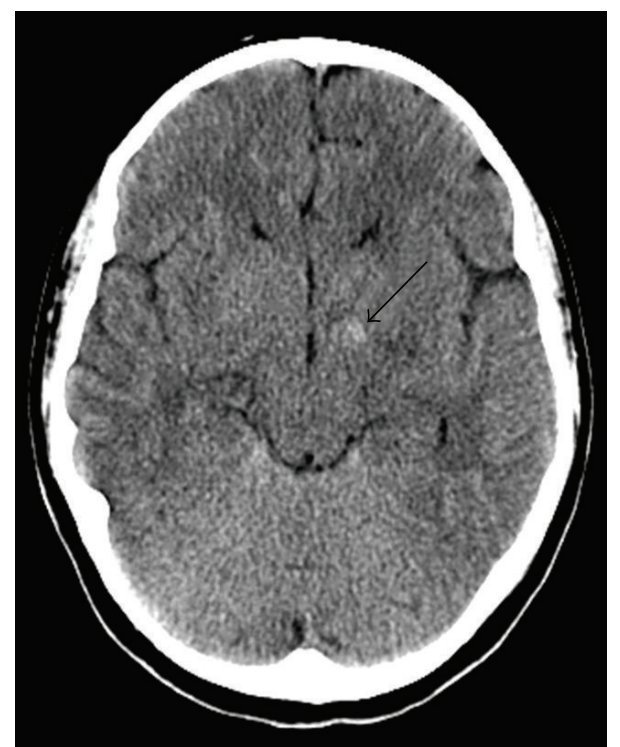

Figure 7: A small, well-defined hyperdense lesion in the left frontal basal area, just below the basal nuclei, is recognizable at the emergency CT (arrow). There is no evidence of subarachnoid haemorrhage.

a dramatic clinical improvement, and neurological examination demonstrated only a mild right cerebellar syndrome. Repeated transoesophageal echocardiography during Valsalva maneuver revealed an atrial septal defect that was repaired via percutaneous endovascular approach due to significant resting shunts. Clinical and neuroradiological followups since then were unremarkable.

7.3. Case 3 (Figures 5 and 6). A 4-year-old female, with a strong family history (father and grandfather) of hereditary hemorrhagic telangiectasia (HHT) or Rendu-Osler-Weber disease, was first evaluated at our institute at the age of
3 months for left temporal lobe hemorrhagic lesions detected elsewhere in the neonatal period. Neonatal history was complicated by tremors and generalized seizures that started on the second day of life after normal pregnancy and delivery.

On first admission at our institute, she was severely hypotonic; epilepsy was well controlled by antiepileptic monotherapy. Serial brain MRI and MRA studies (Figure 5) revealed left cortical-subcortical temporo-parietal-occipital gliotic-malacic areas associated with a cortical malformation.

A possible vascular malformation was also suspected in the left temporal hemisphere. Due to the age and the clinical condition of the patient, DSA was not performed at that time.

The patient presented psychomotor and language delay, and at the age of 4 years she started having recurrent episodes of epistaxis. Genetic analysis confirmed the HHT diagnosis with a mutation in the endoglin gene (ENG) on chromosome 9. Abdominal and pulmonary artero-venous malformations were excluded after adequate screening.

Four vessels DSA was then performed under general anesthesia, showing a left temporal arteriovenous shunt with an abnormal vascular dilatation, probably consistent with a venous varix (Figure 6(a)). It was successfully treated via endovascular approach with a superselective catheterization of the left anterior temporal artery, obliterating the venous varix and the shunt, utilizing detachable platinum coils and histoacrylic glue (Figure 6(b)).

7.4. Case 4 (Figures 7 and 8). A 12-year-old girl, who complained of a sudden headache with no other neurological deficit, was brought to the Emergency Room of another hospital. CT scan showed a small, round area of hyperdensity in the left basal frontal lobe, just below the basal ganglia (arrow in Figure 7). No subarachnoid blood was depicted. The patient was referred to our hospital for further examinations. At admittance, the patient was awake, well oriented, with no neurological deficit, but suffering 


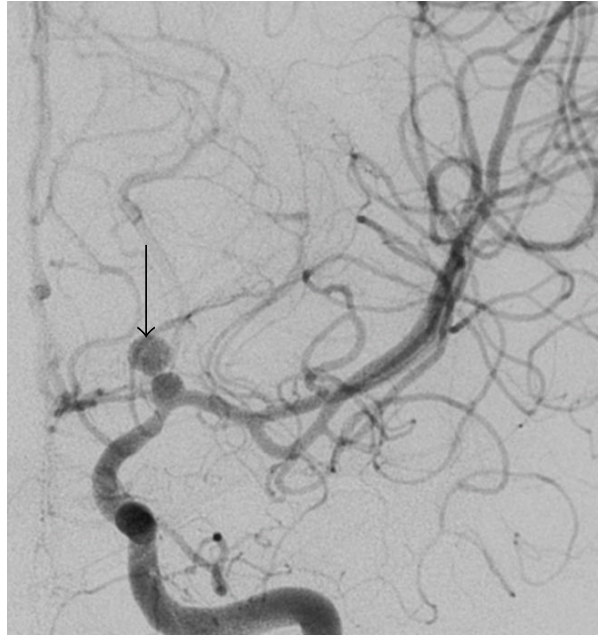

(a)

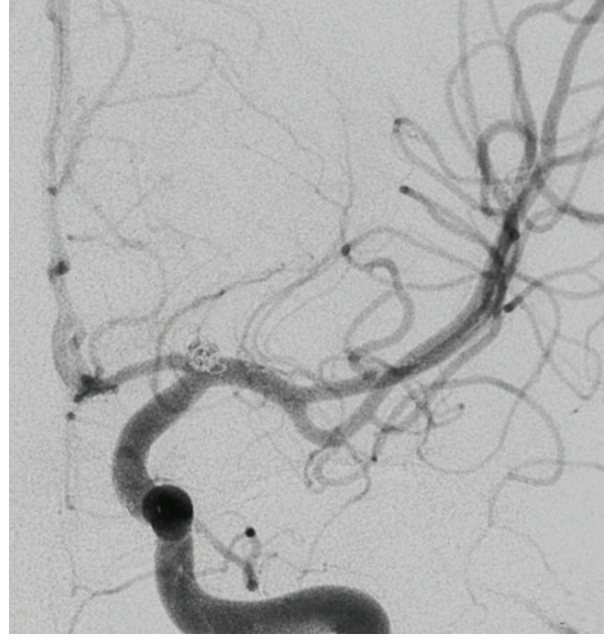

(b)

FIGURE 8: Anteroposterior view of the left internal carotid artery angiography shows a blister-like aneurysm at the intracranial bifurcation (a). Note the small pseudoaneurysm (arrow in (a)) at the dome of the aneurysmal sac, corresponding to the image seen on the CT (Figure 7), and the spasm in the supraclinoid portion of the carotid artery. The posttreatment angiography shows successful obliteration of the aneurysm with stent and a single platinum coil (b).

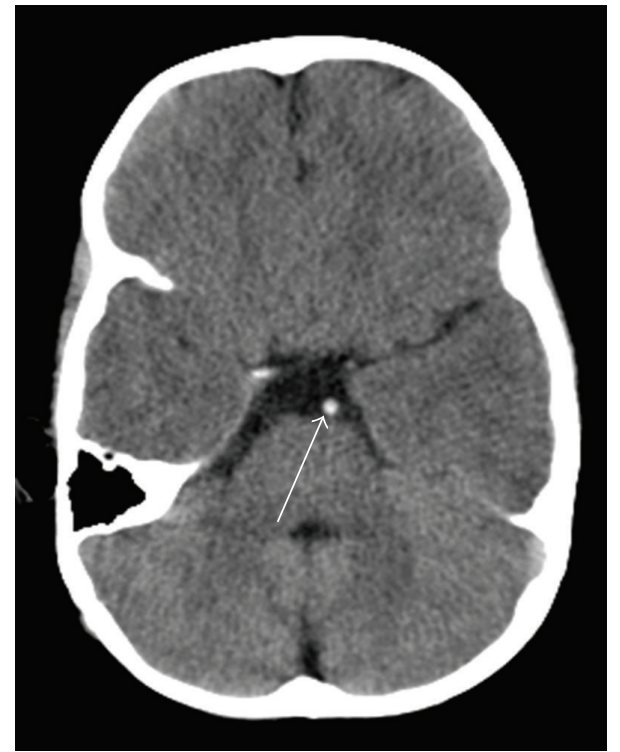

Figure 9: CT at admittance to Emergency Room shows a hyperdensity of the basilar artery, consistent with a thrombus (arrow).

from intense headache. DSA showed a small "blister-like" aneurysm at the left internal carotid artery bifurcation with a pseudoaneurysm just above it (arrow in Figure 8(a)). The patient was treated by endovascular approach, considering the neurosurgical approach more risky due to the presence of many perforating vessels in the area. Since the neck of the aneurysm was wide, a neuroform stent was deployed and a single platinum coil detached after passing through the struts of the stent. The final control showed the occlusion of the sac and the normal flow in the carotid artery (Figure 8(b)), with contrast stagnation inside the pseudoaneurysm. After

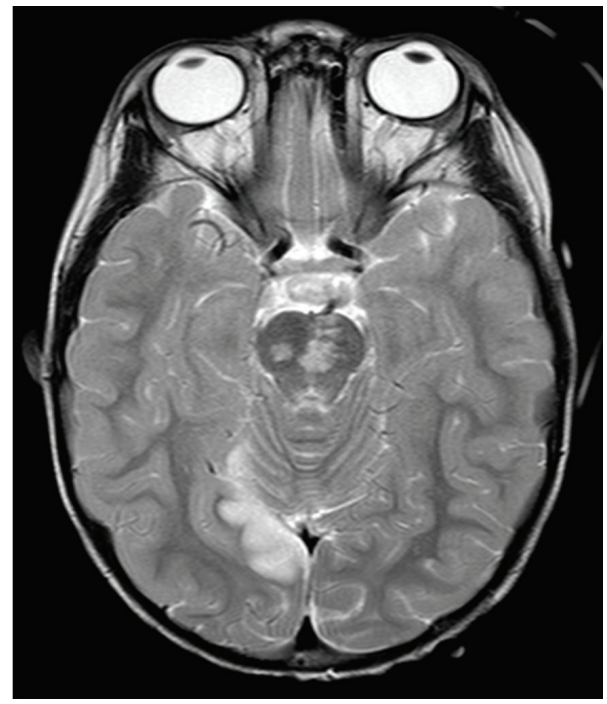

FIGURE 10: The axial T2 MR study performed 3 days after symptoms onset demonstrates the ischemic lesions in the pons and right occipital cortex.

the stent deployment, antiplatelet therapy was started and kept for 6 weeks. The patient had no deficit. Headache progressively decreased in the following days.

No recurrence of the aneurysm was observed at the 6month follow-up angiography.

7.5. Case 5 (Figures 9-11). A 3-year-old African boy presented with sudden onset of nausea and vomiting. He was admitted to the Emergency Room hypotonic, hyporeactive, with abnormal ocular movements and recurrent seizures. In the recent past medical history, only sporadic headache was 
reported, after a varicella infection. At brain CT a hyperdense basilar artery was visible (arrow in Figure 9). At MRI with MRA, ischemic lesions in the vertebrobasilar territory with severe obstruction of the basilar and right posterior cerebral arteries were observed. As some hours had already passed from symptoms onset and the experience as well as the literature data on thrombolysis in children were poor, it was decided not to proceed to thrombolysis neither by venous nor arterial route. In addition, clinical conditions, though severe, did not seem to be life-threatening at that moment. Over the next few days, patient clinical conditions slowly but progressively improved. An MRI control at day 3 showed a new lesion in the pons (Figure 10). A DSA performed at day 12 confirmed the MRI findings: occlusion of the basilar artery from the vertebrobasilar (VB) junction to the tip, with good collaterals supplying the brainstem perforators and partial revascularization of the superior cerebellar arteries (arrows in Figure 11).

In the diagnostic workup, a patency of the foramen ovale was found, as the cardiologists did not consider the thromboembolic source causing the occlusion. At day 22, at discharge, no neurological deficits were present except for a very slight difficulty in walking with a preferred use of the left limbs.

\section{Discussion and Conclusion}

Stroke in paediatric population is associated with relative high morbidity, mortality, and recurrence rates. Silent stroke can be responsible for neuropsychological disability not otherwise recognizable.

If a stroke is suspected in a child, the diagnostic workup should include systemic blood tests (haematological disorders, infections), genetic and cardiac examinations (see our case 2). The neuroradiological diagnosis comprehends CT scan, MR imaging, and DSA. CT scan may demonstrate the presence and site of the intracranial bleeding (SAH or intracerebral haematoma) in case of HS. MR imaging with MRA better delineates the brain tissue status, the presence, the nature, and sometime the timing of possible structural lesions, for both HS and IS. They represent crucial tools both for treatment planning and followup. DSA is an invasive exam that should be performed by expert operators and only in selected cases. MRI and DSA in childhood are commonly performed in general anaesthesia to ensure a good quality imaging without any discomfort for the young patient.

In the appropriate setting, besides medical treatment, invasive procedures including endovascular approach should be considered. In cases of structural lesions a thorough risk/benefit analysis in order to decide the best treatment option is mandatory. Timing of invasive treatment can also represent a troublesome factor in a very young patient in whom fluid balance is a critical aspect in the anesthesiologi$\mathrm{cal}$ and surgical management. In very small children, for this reason, it may be necessary to delay treatment waiting for a more favourable clinical status such as in our case 3.

In case of aneurysm the endovascular approach includes the obliteration of the fundus utilizing detachable coils associated with an intracranial stent in case of large neck,

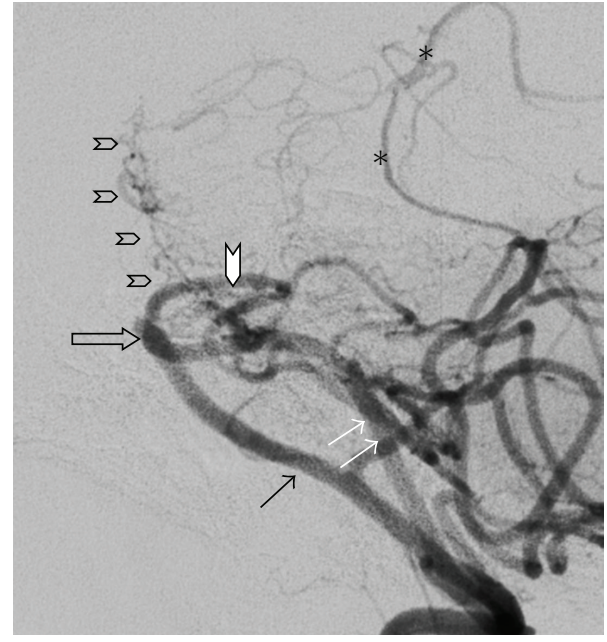

FIGURE 11: Cerebral angiography (lateral view) with injection of the left vertebral artery (arrow) shows occlusion of the basilar artery (open arrow) at the vertebrobasilar junction, just above the origin of the right superior cerebellar artery (white arrowhead). There are many tiny collaterals that supply the territory of the brainstem perforators (small arrowheads) and wider collaterals that reconstitute the superior cerebellar artery (asterisks). There is also retrograde filling of the right vertebral artery (white arrows) and of the right PICA.

such as in our case 4. Experience in stent placement in children is limited; double antiplatelet therapy in case of stent deployment is recommended; since there are not specific trials for antiplatelet treatment for intracranial stenting in children, medical treatment is reasonably based on the adult's literature data. Another concern with paediatric intracranial stenting is the ongoing growth of the vessels over the time $[11,12,23]$.

Dissecting aneurysm represents a typical vascular malformation in which the treatment must be tailored case by case. In case of a large dissecting aneurysm in a distal vascular territory, the obliteration of the arterial feeder with coils or glue should be taken into consideration. A balloon occlusion test to verify the possible collateral network can be previously performed. In case of a large thrombotic dissecting aneurysm in a vital territory, a precise preoperatory planning should be carried out, balancing benefits and risks. In our case 1, where a nonruptured large partially thrombosed aneurysm of the basilar artery causes a critical stenosis of the vessel, we preferred to delay the management of the aneurysm and of the BA stenosis because of the good clinical condition of the patient. Therefore, we followed the patient with MRI and MRA every 2-4 months. The spontaneous occlusion of the BA with repair of the aneurysm occurred asymptomatically.

Detachable coils and glue can be successfully utilized to occlude arteriovenous fistulas or AVM, such as in our case 3 in whom the direct AV fistula was related to HHT; in fact the presence of a cerebral AVM is one of the four diagnostic criteria for HHT [27]. 
In childhood, revascularization procedures in thrombotic vessels utilizing an endovascular approach are anecdotic (our case 5), likewise for the new flow-diverters devices that are indicated for aneurysmal repair in adult, and no literature data or specific recommendations are available as already discussed in the Treatment section.

In conclusion, stroke in childhood represents a challenging disease in which a correct diagnostic conduct may lead to the most appropriate treatment. A decision-making process should be carried out case by case by a multispecialist group, including the neuropaediatrician, neurosurgeon, and neuroradiologist. Future trials focused on treatment of vascular diseases in the youngest population are necessary to better understand the disease and, therefore, to improve the treatment.

\section{Conflict of Interests}

The authors declare that they have no conflict of interests.

\section{References}

[1] L. A. Beslow and L. C. Jordan, "Pediatric stroke: the importance of cerebral arteriopathy and vascular malformations," Child's Nervous System, vol. 26, no. 10, pp. 1263-1273, 2010.

[2] L. C. Jordan, "Assessment and treatment of stroke in children," Current Treatment Options in Neurology, vol. 10, no. 6, pp. 399-409, 2008.

[3] A. A. Mallick and F. J. K O'Callaghan, "Risk factors and treatment outcomes of childhood stroke," Expert Review of Neurotherapeutics, vol. 10, no. 8, pp. 1331-1346, 2010.

[4] T. Krings and I. S. Choi, "The many faces of intracranial arterial dissections," Interventional Neuroradiology, vol. 16, no. 2, pp. 151-160, 2010.

[5] C. Amlie-Lefond, T. J. Bernard, G. Sebire et al., "Predictors of cerebral arteriopathy in children with arterial ischemic stroke: results of the international pediatric stroke study," Circulation, vol. 119, no. 10, pp. 1417-1423, 2009.

[6] H. J. Cho, Y. H. Jung, Y. D. Kim, H. S. Nam, D. S. Kim, and J. D. Heo, "The different infarct patterns between adulthoodonset and childhood-onset moyamoya disease," Journal of Neurology, Neurosurgery \& Psychiatry, vol. 82, no. 1, pp. 3840, 2011.

[7] D. M. Ibrahimi, R. J. Tamargo, and E. Ahn, "Moyamoya disease in children," Child's Nervous System, vol. 26, no. 10, pp. 1297-1308, 2010.

[8] D. Kenny, M. Turner, and R. Martin, "When to close a patent foramen ovale," Archives of Disease in Childhood, vol. 93, no. 3, pp. 255-259, 2008.

[9] C. Tzoulis and L. A. Bindoff, "Serial diffusion imaging in a case of mitochondrial encephalomyopathy, lactic acidosis, and stroke-like episodes," Stroke, vol. 40, no. 2, pp. e15-e17, 2008.

[10] N. Diamini, L. Billinghurst, and F. J. Kirkham, "Cerebral venous sinus (sinovenous) thrombosis in children," Neurosurgery Clinics of North America, vol. 21, no. 3-5, pp. 511-527, 2010.

[11] T. Krings, S. Geibprasert, and K. TerBrugge, "Classification and endovascular management of pediatric cerebral vascular malformations," Neurosurgery Clinics of North America, vol. 21, no. 3, pp. 463-482, 2010.
[12] T. Krings, S. Geibprasert, and K. G. TerBrugge, "Pathomechanisms and treatment of pediatric aneurysms," Child's Nervous System, vol. 26, no. 10, pp. 1309-1318, 2010.

[13] L. C. Jordan, S. C. Johnston, Y. W. Wu, S. Sidney, and H. J. Fullerton, "The importance of cerebral aneurysms in childhood hemorrhagic stroke a population-based study," Stroke, vol. 40, no. 2, pp. 400-405, 2009.

[14] H. J. Fullerton, Y. W. Wu, S. Sidney, and S. C. Johnston, "Risk of recurrent childhood arterial ischemic stroke in a population-based cohort: the importance of cerebrovascular imaging," Pediatrics, vol. 119, no. 3, pp. 495-501, 2007.

[15] Paediatric Stroke Working Group, "Stroke in childhood: clinical guidelines for diagnosis, management and rehabilitation," 2004.

[16] A. Kirton, J. H. Wong, J. Mah et al., "Successful endovascular therapy for acute basilar thrombosis in an adolescent," Pediatrics, vol. 112, no. 3, pp. e248-e251, 2003.

[17] J. Irazuzta and K. J. Sullivan, "Hyperacute therapies for childhood stroke: a case report and review of the literature," Neurology Research International, vol. 2010, Article ID 497326, 2010.

[18] H. J. Fullerton, Y. W. Wu, S. Sidney, and S. C. Johnston, "Recurrent hemorrhagic stroke in children: a populationbased cohort study," Stroke, vol. 38, no. 10, pp. 2658-2662, 2007.

[19] P. Monagle, E. Chalmers, A. Chan et al., "Antithrombotic therapy in neonates and children: american college of chest physicians evidence-based clinical practice guidelines (8th edition)," Chest, vol. 133, no. 6, pp. 887S-968S, 2008.

[20] E. S. Roach, M. R. Golomb, and R. Adams, "Management of stroke in infants and children: a scientific statement from a special writing group of the american heart association stroke council and the council of cardiovascular disease in the young," Stroke, vol. 39, no. 9, pp. 2644-2691, 2008.

[21] S. Grigoriadis, J. M. Gomori, N. Grigoriadis, and J. E. Cohen, "Clinically successful late recanalization of basilar artery occlusion in childhood: what are the odds? Case report and review of the literature," Journal of the Neurological Sciences, vol. 260, no. 1-2, pp. 256-260, 2007.

[22] Y. Y. Lee, K. L. Lin, H. S. Wang et al., "Craniocervical arterial dissection: a cause of childhood arterial ischemic stroke in Taiwan," Journal of the Formosan Medical Association, vol. 109, no. 2, pp. 156-162, 2010.

[23] Y. J. Lai, F. C. Chang, C. J. Lin, T. C. Hsieh, and K. L. Wang, "Endovascular therapy in pediatric intracranial carotid artery dissection," Pediatric Neurology, vol. 42, no. 4, pp. 291-294, 2010.

[24] R. Gupta, H. J. Yi, T. G. Jovin, and M. B. Horowitz, "Three unusual arteriovenous fistulas presenting in young children successfully treated with transarterial endovascular embolization," Pediatric Neurosurgery, vol. 41, no. 6, pp. 328333, 2005.

[25] E. F. Ciceri, C. Regna-Gladin, A. Erbetta et al., "Iatrogenic intracranial pseudoaneurysms: neuroradiological and therapeutical considerations, including endovascular options," Neurological Sciences, vol. 27, no. 5, pp. 317-322, 2006.

[26] V. K. Sharma, H. L. Teoh, L. Y. Wong, J. Su, B. K. Ong, and B. P. Chan, "Recanalization therapies in acute ischemic stroke: pharmacological agents, devices, and combinations," Stroke Research and Treatment. In press.

[27] T. Krings, S. M. Chng, A. Ozanne, H. Alvarez, G. Rodesch, and P. L. Lasjaunias, "Hereditary hemorrhagic telangiectasia in children: endovascular treatment of neurovascular malformations," Neuroradiology, vol. 47, no. 12, pp. 946-954, 2005. 
[28] D. Kondziolka, H. Kano, H. C. Yang, J. C. Flickinger, and L. Lunsford, "Radiosurgical management of pediatric arteriovenous malformations," Child's Nervous System, vol. 26, no. 10, pp. 1359-1366, 2010. 


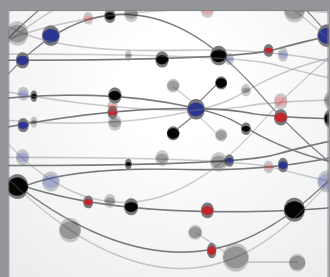

The Scientific World Journal
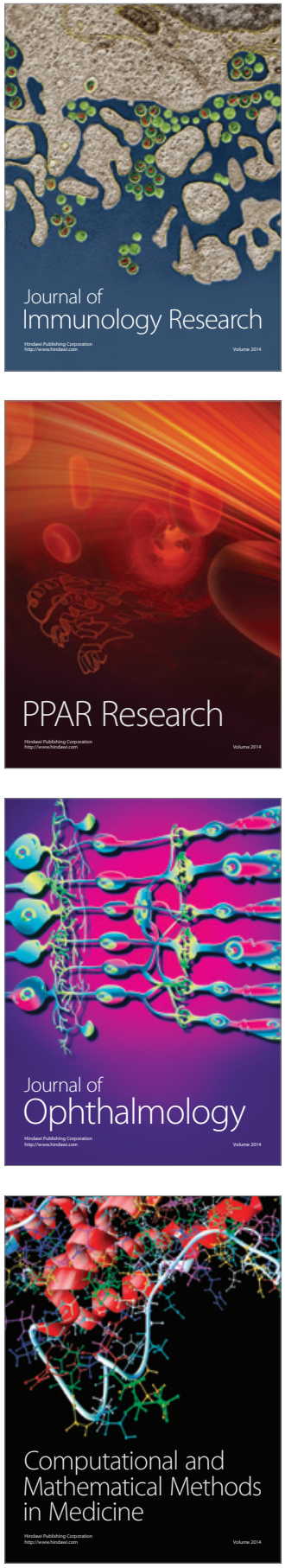

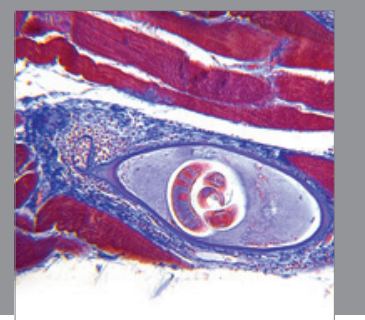

Gastroenterology

Research and Practice
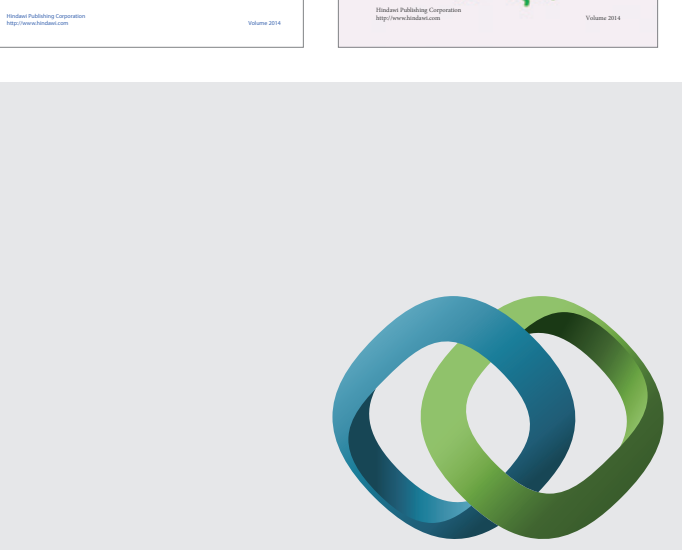

\section{Hindawi}

Submit your manuscripts at

http://www.hindawi.com
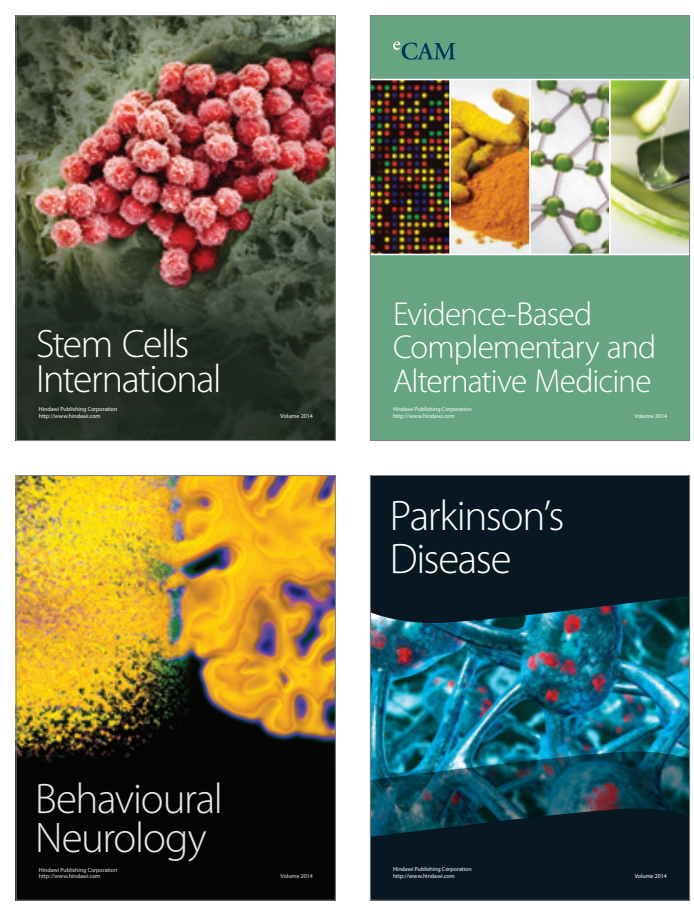

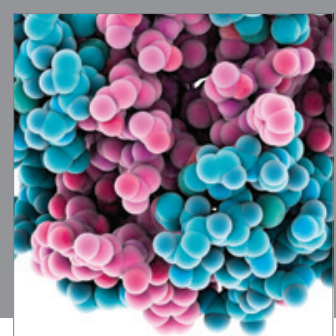

Journal of
Diabetes Research

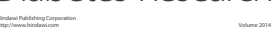

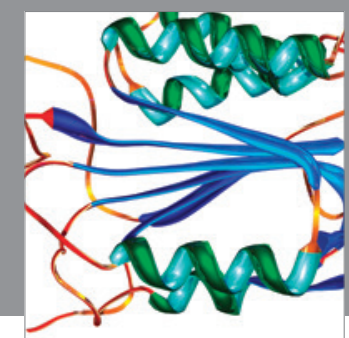

Disease Markers
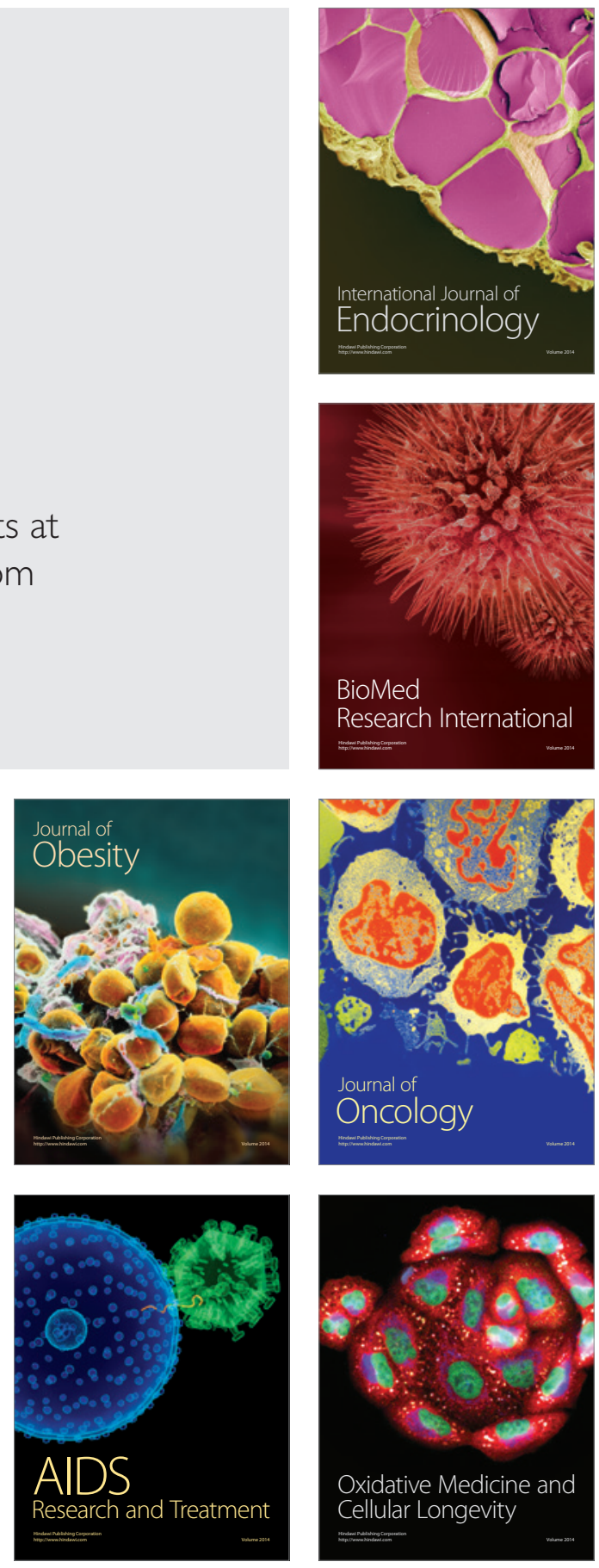